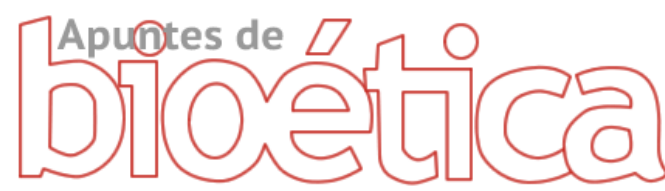

https://doi.org/10.35383/apuntes.v2i1.243

\title{
La Inclusión de la Bioética como eje transversal en la EBR
}

\author{
José Rogelio Ruiz Alvarado ${ }^{1}$
}

\begin{tabular}{l}
\hline INFORMACIÓN DEL ARTÍCULO \\
\hline Historia del artículo: \\
Recibido el 21 de marzo de 2019 \\
Aceptado el 25 de julio de 2019 \\
\hline
\end{tabular}

claves:

Inclusión

Eje transversal

Bioética

Educación Básica Regular

\section{RESUMEN}

La sociedad peruana, enfrenta situaciones en que las personas manifiestan humillación por la vida humana sin ningún tipo de remordimiento; es posible descubrir que la gran mayoría de veces, los autores de estos actos lesivos provienen de hogares disfuncionales, de un ambiente de violencia familiar, e incluso, muchos de ellos no terminaron la educación básica regular, nos refiriendo al proceso que requiere compromiso y entrega, no solo de parte de los docentes, sino también de los padres o tutores, quienes reforzarán en casa lo que a sus hijos o pupilos se les enseñe en las instituciones educativas. El importante papel que juega la educación, no lo es sólo en el aspecto de avances o descubrimientos científicos y tecnológicos, sino de calidad humana de las personas, a sus principios éticos, bioéticos y sus valores morales que se aprenden durante la etapa escolar. El ámbito de acción de la bioética se ha amplificado, hoy en día, abarca no solo la ética médica, la ética ambiental, los debates sobre los derechos de las futuras generaciones, el desarrollo sostenible, etc. Para incluir el eje transversal de la bioética en la educación, se tendrán que tomar en cuenta la parte que conforma la esencia de la bioética, sus principios y la humanización de la educación.

\section{The Inclusion of Bioethics as a Transverse Axis in the EBR}

\section{ABSTRACT}

Keywords.

Inclusion

Transverse Axis

Bioethics

Regular Basic Education
The Peruvian society, faced situations in which people express humiliation for life without any remorse; It is possible to discover the vast majority of times, the authors of these harmful acts come from dysfunctional homes, an atmosphere of family violence, and even many of them did not finish the basic regular education, we are referring to the process that requires commitment and delivery, not only on the part of teachers, but also of parents or guardians, who will reinforce at home what you teach your children or wards in educational institutions. The important role played by education, it is not only in the aspect of scientific and technological discoveries and advances, but human quality of people, to its ethical

${ }^{1}$ Licenciado en Ciencias Históricas Sociales y Filosofía, Segunda Especialidad en Psicopedagogía, Maestro en Docencia y Gestión Educativa, estudios en Bioética y Biojurídica, Docente en Educación Básica Regular de la Institución Educativa Santa Lucía - Ferreñafe, Docente de Pregrado de la Universidad Católica Santo Toribio de Mogrovejo. Chiclayo, Perú. Email: joserogelio_ruiz@hotmail.com. ORCID: https://orcid.org/0000-0003-1683-2787 
principles, ethical and moral values that are learned during the school. The scope of bioethics has amplified, today, includes not only the medical ethics, environmental ethics, debates about the rights of future generations, sustainable development, etc. To include the transverse axis of the bioethics education, they will have to take into account the part that makes up the essence of bioethics, its principles and the humanisation of education.

\section{Introducción}

Todos en algún momento hemos escuchado la famosa frase "se educa con el ejemplo", y no hay nada más cierto que eso. La educación que brindan los maestros a los educandos, desde los primeros años de formación educativa es la principal fuente de aprendizaje para ellos. Los maestros son el modelo a seguir de sus alumnos, ellos les enseñan no solo las experiencias curriculares sino también, valores, formas de comportarse, normas para una buena convivencia, les enseñan a compartir, a cuidar el medio ambiente, a proteger a los animales, entre otras cosas más; entonces, por qué no incluir en este proceso educacional, dentro de la enseñanza y la instrucción que se brinda a los estudiantes, la formación en bioética como un eje transversal en los planes educativos.

\section{Bioetica, réplica a las dificultades y circunstancias en Educación Básica Regular.}

La sociedad peruana, viene enfrentando en las últimas décadas situaciones realmente espantosas, tenemos escenas en que las personas actúan manifestando un total desprecio por la vida humana sin ningún tipo de remordimiento, actos delincuenciales en los que sorprendente el valor de la vida se equipara, por ejemplo, al de un teléfono móvil; somos testigos a través de los medios de comunicación de crímenes cometidos por hijos en contra de sus padres simplemente por una motivación lucrativa, asesinatos cometidos contra mujeres y niños, éstas son solo algunas de las manifestaciones de la crisis social a la cual enfrentamos. Esta situación nos lleva a preguntarnos ¿por qué?, ¿dónde radica? o ¿de qué depende?; al realizar un análisis a grosso modo, nos será posible descubrir que la gran mayoría de veces, las personas que se convierten en los autores de estos actos lesivos provienen de hogares disfuncionales, crecieron dentro de un ambiente de violencia familiar, e incluso, muchos de ellos no terminaron la educación básica regular. Si bien podemos afirmar que ya existen programas nacionales y sociales que promueven nuestros gobernantes $y$ representantes para luchar contra ciertas situaciones que dan origen a dicha problemática, se ha dejado de lado un aspecto, que podría afirmar, es el más importante y que, de ser utilizado correctamente, será un elemento clave en la lucha contra esta crisis social: la educación.

Al referirnos a la educación, no nos estamos refiriendo a su sentido estricto centrado solo en aquella instrucción de materias básicas y necesarias en las instituciones educativas dentro de su currículo de estudios con cuya aprehensión los educandos lograrán escribir y leer correctamente así como realizar operaciones matemáticas e incluso conocer de historia universal y la composición de los seres vivos; al concepto de educación al que nos estamos refiriendo es el proceso que requiere compromiso y entrega, no solo de parte de los maestros, sino también de los padres, quienes reforzarán en casa lo que a sus hijos se les enseñe en sus centros de estudios.

El hombre es el único ser vivo que necesita aprender a comportarse como lo que es; los demás no lo necesitan, pues ya poseen en su propia dotación genética, en su naturaleza, las 
pautas fijas y estereotipadas respecto a su conducta. Pero el hombre no es sólo genético, sino también cultura, la misma que forma precisamente a partir de la educación.

El recibir de los demás, en la primera instancia de los padres o tutores es lo necesario para comenzar a conducirse en la vida como lo que es y se debe ser. Por eso el hombre es un ser social por naturaleza. Su precariedad biológica, por otra parte, hace necesario acudir al factor cultural para dar cuenta, no sólo de su viabilidad sino también de su superioridad sobre todo el orden animal.

La llamada transversalidad llegó a su máxima expresión después de que se publicó el denominado "Informe de la Comisión Internacional sobre la Educación para el Siglo XXI", difundido por la UNESCO en 1996. A partir de ese suceso en adelante, algunas instituciones han adoptado este instrumento en sus diseños curriculares incluyendo temas que cumplen el objetivo de tener una visión de conjunto (UNESCO, 2006).

Entonces podemos decir que, los ejes transversales se constituyen en fundamentos para la práctica pedagógica, al integrar los campos del ser, el saber, el hacer y el convivir, mediante conceptos, procedimientos, valores $y$ actitudes que orientan la enseñanza y el aprendizaje. Asimismo, es necesario aclarar que el hecho de agregar a la educación un enfoque transversal no significa desplazar la importante labor de las disciplinas propias de la enseñanza escolar; sin embargo, su inclusión en ella obligará a que los mecanismos y medios utilizados de forma tradicional en las aulas puedan ser verificados y adaptarlos en cada uno de sus niveles buscando así que, la educación que se les brinde a los estudiantes sea significativa y contemple una conexión entre sus materias y los problemas sociales, problemas éticos e incluso morales que puedan estar aquejando contexto y ámbito en que se desarrolla y del cual es parte (Argueta, 2004).

Es menester aclarar que esta formación en bioética estará orientada ampliamente a inculcar un sentido de respeto por la vida humana en todo tipo de contextos y situaciones en los estudiantes a través de la educación. Sería imposible superar la crisis actual de nuestra sociedad, en cuanto al respeto por la vida se refiere, si no que se instruye y educa desde la niñez que todo aquello que atente contra la vida está mal, y que de ninguna manera podrán justificarse los atentados en su contra. Ese es precisamente el motivo del presente artículo, asegurando que la mejor manera para lograr una consciencia de respeto y valoración suprema de la vida es iniciando desde la educación; todo, absolutamente todo lo que se nos enseña en las escuelas, estará siempre presente en nuestras vidas y por ello es que se debe incluir la bioética como eje transversal en el proceso educativo.

Quienes nos representan, así como quienes nos gobiernan, están siempre buscando formas de poder combatir, luchar, disminuir o erradicar los diversos problemas que enfrenta nuestra sociedad; y han dejado en el olvido a la que podría convertirse en su mayor arma en esta constante lucha, la educación. El importante papel que juega la educación, no lo es sólo en el aspecto de avances o descubrimientos científicos y tecnológicos, sino en cuanto a la calidad humana de las personas, a sus principios éticos y sus valores morales que se aprenden durante los primeros años de vida.

Al incluir la bioética en los niveles de Educación Básica Regular será necesario tomar en cuenta el Proyecto Educativo Nacional como Política de Estado (Botero, 2006) y cuyos objetivos apuntan al año 2021, que busca lograr un cambio educativo como instrumento orientador de elementos significativos y trascendentales: 
1. Una educación con equidad, que asegure oportunidades y resultados educativos de calidad a todos los peruanos y sin discriminaciones.

2. Una educación de calidad, que haga de las escuelas, instituciones eficaces e innovadoras, que enseñen bien $y$ que garanticen que todos aprendan.

3. Buenos docentes, con oportunidades de desarrollo profesional que lo ayuden a desempeñarse mejor cada vez y a ser reconocido social y laboralmente por su buen desempeño.

4. Una gestión educativa ética, democrática y eficaz, con financiamiento suficiente para los cambios que se necesitan.

5. Una educación superior renovada, que aporte nuevos conocimientos al desarrollo del país y a su inserción competitiva en la economía mundial.

6. Una sociedad educadora, que fomente el compromiso de todas las comunidades con la formación de ciudadanos identificados con su propia localidad.

Además de los mencionados se debería agregar en cada uno de ellos el enfoque de la bioética que se convertirá en parte del proceso educativo, del cual se esperará obtener un futuro mejor, no solo política y socialmente, sino también en el sentido de humanidad que tendrán los ciudadanos (De Los Ríos, 2003).

En este caminar, el objetivo parte de aprender a vivir con principios bioéticos sólidos que reconozcan que las personas tenemos derechos y responsabilidades basados en principios morales a través del desarrollo de competencias, para ello se requerirá de un trabajo conjunto que englobe el esfuerzo personal, el afán de superación, la dedicación y la autodisciplina, así como el respeto y la tolerancia, la generosidad y la solidaridad (Padovani, 2010).

La propuesta podría quedarse solo en el idealismo si no se realiza un análisis de la realidad a la que nos enfrentamos en el sector educación, es necesario considerar que aún existe un vacío en la Formación Bioética en el profesional que ejerce la docencia. Es aquí donde tenemos el primer y más importante paso para la inserción de la formación bioética en el proceso educacional, la capacitación y actualización de docentes, puesto que serán ellos quienes llevarán directamente la ejecución de este cambio, por lo tanto, son ellos los primeros que deben tener en su mentalidad que todo lo que trasmitan a sus estudiantes deberán tener la connotación bioética.

Generalmente, al escuchar la palabra bioética, las personas pueden imaginarse que se hace referencia a una ciencia que únicamente los profesionales de la salud deben conocer y aplicar, sin embargo, la bioética ocupa un espacio en la mayoría de contextos en los cuales las personas nos podremos desenvolver profesional y socialmente.

Ahora veamos, cuál será la parte inicial de la bioética que se insertará en el proceso de educación.

Conforme la bioética ha ido evolucionando, su ámbito de acción también se ha amplificado, hoy en día, la bioética abarca los aspectos tradicionales de la ética médica, la ética ambiental, los debates sobre los derechos de las futuras generaciones, el desarrollo sostenible, etc. Como podemos ver, la bioética es una instancia de juicio práctico que se ejerce en circunstancias concretas y a la que se le asigna 
una finalidad práctica a través de diferentes formas de institucionalización (Zuleta, 2017).

Así tenemos que, la bioética debe concebirse en el contexto interdisciplinario donde los profesionales buscan humanizar las áreas del saber, como un movimiento social y cultural de los ciudadanos, es a su vez un área de conocimiento que se refiere a la moralidad de las nuevas formas de nacer, vivir, curar, cuidar y morir. Para incluir el eje transversal de la bioética en la educación, se tendrán que tomar en cuenta la parte que conforma la esencia de la bioética, sus principios, los cuales detallaremos a continuación:

- Principio de Autonomía. Este principio se refiere a la necesidad de respetar, a través de nuestras acciones y actitudes, la capacidad y el derecho que poseen las personas para que puedan libremente decidir entre las opciones que a su juicio son las que mejor resultado podrían darles después de compararlas con las diferentes posibilidades de las que se les haya informado, conforme a sus valores, creencias y planes de vida. Este principio se manifestará en decisiones respecto a su cuerpo y a su salud, inclusive en términos de intervenciones como de investigación.

- Principio de Beneficencia. De acuerdo a lo que implica este principio, se deberán hacer evaluaciones sobre posibilidades de las ventajas y las desventajas, los riesgos y los beneficios que puedan suponer los procesos de los que formará parte, o de los procedimientos de investigación, con el objeto de maximizar los beneficios y disminuir los riesgos. Este principio contiene también una dimensión positiva que implica el deber inquebrantable de llevar a cabo acciones específicas encaminadas a procurar el bienestar de las personas, defender sus derechos, prevenir el daño, eliminar las condiciones que le generan riesgo, malestar y dolor, entre otras.

- Principio de No Maleficencia. Atendiendo a este principio, tenemos que, no se debe infligir daño o hacer mal. Por este principio es que las personas están obligadas a evitar el daño físico o emocional y el perjuicio de las demás personas.

- Principio de Justicia: Este principio será uno de los más importantes que se deberán transmitir, por medio de él se pretende que la distribución de los beneficios, los riesgos y los costos se realicen en forma justa. Es decir, que se distribuyan equitativamente entre todos los grupos de la sociedad, tomando en cuenta la edad, el sexo, el estado económico y cultural, y consideraciones étnicas.

La bioética tiene un sentido común para todos que es el actuar con la recta razón de día a día ayudando a encontrar un sentido real a la vida, en medio de este mundo pragmático, hedonista, reduccionista, materialista y que no cree ya en nada. Razón por la cual no se puede obviar los principios de la bioética personalista y que gracias a ella se fundamenta en el ser de la persona humana y en su dignidad, modelo en el cual se mantiene el primado y la intangibilidad de la persona humana, considerado como valor supremo, punto de referencia, fin y no medio, permitiendo construir una bioética plenamente respetuosa de la dignidad ultima de la persona humana (Campos, 2019).

- Principio de defensa de la vida física: subraya la corpórea, como esencia de la persona, enfatizando el derecho a la vida y a la integridad física.

- Principio de Totalidad: basada en el bien y recuperación de la persona humana, en la 
búsqueda de los medios lícitos para sanar el cuerpo.

- Principio de Libertad y Responsabilidad: Destaca la esencia de la persona como ser libre para alcanzar lo bueno y lo justo ante su propia vida, los demás y entorno.

- Principio de sociabilidad y de solidaridad: recalca la existencia y el respeto de la persona como bien social y el fomento del bien común

\section{Metodología}

La investigación responde a la naturaleza cuantitativa - cualitativa, expresada en el método inductivo, valiéndose de la reflexión y las premisas lógicas que convergen en las conclusiones de hechos específicos, iniciándose con la disertación individual que postula el fundamento de la bioética.

En lo referente a lo cualitativo, se orienta a profundizar casos específicos y no generalizados, basado en cualificar y describir la formación bioética de los docentes de Educación Básica Regular partir del desarrollo de los contenidos transversales con contenidos bioéticos.

\section{Conclusiones}

Entonces podemos decir que, los ejes transversales se constituyen en fundamentos para la práctica pedagógica, al integrar los campos del ser, el saber, el hacer y el convivir, mediante conceptos, procedimientos, valores y actitudes que orientan la enseñanza y el aprendizaje.

Ante las situaciones problemáticas la bioetica es una respuesta a las dificultades y circunstancias que se presenta en nuestro entorno que permitan suscitar el análisis y reflexión que presentan el entorno educativo, enmarcados en las actitudes y valores como base sus disertaciones procedentes y tomadas libremente.

La inclusión de la bioética como eje transversal en la educación, favorecerá a la formación de personas capaces de ser seres responsables y autónomos en sus enjuiciando de manera crítica ante sus dimensiones, físicas, antropológicas y éticas y los diferentes ámbitos que se desarrolla como ser humano y la persona.

La educación es un proceso propio del hombre, que se da en muchos ambientes, pero de manera formal en las instituciones educativas. La educación consiste en un proceso de toda la vida (no sólo la niñez y la juventud) con actividades que permiten formar valores $y$ virtudes, transformar conductas y poner en acción las representaciones mentales para intervenir en el mundo circundante, $y$ es precisamente por ese motivo que es el instrumento perfecto para incluir la formación en Bioética en nuestra sociedad, que cada vez nos manifiesta con más fuerza la falta que nos hace recobrar el respeto por la vida humana.

\section{Bibliografía}

Argueta, M. (2004). Ejes Transversales en el Currículo de la Formación Inicial de Docentes. COORDINACIÓN EDUCATIVA Y CULTURAL CENTROAMERICANA. Disponible en

http://unpan1.un.org/intradoc/groups/publi c/documents/icap/unpan039733.pdf

Bandrés, M. Perú, Bioética y Medicina, su Enseñanza: Apuntes de una Realidad. Disponible en http://sisbib.unmsm.edu.pe/bvrevistas/spmi /v15n3/bioetica_peru.htm 
Botero, C. (2006). Los ejes transversales como instrumento pedagógico para la formación en valores. Politécnica 3era edición. Disponible en: http://132.248.9.34/hevila/Revistapolitecnic a/2006/no3/5.pdf

Concepto y Principios de la Bioética. Comité Institucional de Bioética. Disponible en: http://www2.congreso.gob.pe/sicr/cendocb ib/con4_uibd.nsf/59F0B1DFF4B09FFB05257 D18006D362B/\$FILE/ceboax-0105.pdf

Campos, P. (2019). Bioética General. Tesis para optar el grado de Maestro en Bioética y Biojurídica. Universidad Católica Santo Toribio de Mogrovejo.

De Los Ríos, F. (2003). Bioética y educación: educación para la bioética. Biblioteca Virtual Universal. Disponible en: http://www.juntadeandalucia.es/educacion/ vscripts/wginer/w/rec/3218.pdf

Espejo, A. (2004). Introducción a la Bioética en Sistema Educativo Español Córdoba. Disponible en: http://www.varona.rimed.cu/revista_varona /index. php?option $=$ com_content\&task $=$ vie w\&id $=223 \&$ Itemid $=33$ \&limitstart $=2$

Londoño, S. y otros. (2002). Manual de iniciación pedagógica al pensamiento complejo. Ediciones Jurídicas Colombia p.158

Padovani, A. (2010). ¿Qué es la Bioética? Revista de Ciencias Médicas de Pinar del Río. Disponible en: http://scielo.sld.cu/scielo.php?script=sci_ar ttext\&pid =S1561-31942010000100036
Unesco Organización de la Naciones Unidas para la Educación, la Ciencia y la Cultura. Declaración Universal sobre Bioética y Derechos Humanos. División de ética de las ciencias y de las tecnologías. Sector de ciencias humanas y sociales. 1 , rue Miollis 75732 Paris Cedex 15 - Francia. 2006

Zuleta, G. (2017). Bioética y educación: educación para la bioética. Magisterio. Disponible en https://www.magisterio.com.co/articulo/bio etica-y-educacion-educacion-para-labioetica 